\title{
EVALUATION FORMULAS FOR AN ANALOGUE OF CONDITIONAL ANALYTIC FEYNMAN INTEGRALS OVER A FUNCTION SPACE
}

\author{
Dong Hyun Cho
}

\begin{abstract}
Let $C^{r}[0, t]$ be the function space of the vector-valued continuous paths $x:[0, t] \rightarrow \mathbb{R}^{r}$ and define $X_{t}: C^{r}[0, t] \rightarrow \mathbb{R}^{(n+1) r}$ and $Y_{t}: C^{r}[0, t] \rightarrow \mathbb{R}^{n r}$ by $X_{t}(x)=\left(x\left(t_{0}\right), x\left(t_{1}\right), \ldots, x\left(t_{n-1}\right), x\left(t_{n}\right)\right)$ and $Y_{t}(x)=\left(x\left(t_{0}\right), x\left(t_{1}\right), \ldots, x\left(t_{n-1}\right)\right)$, respectively, where $0=t_{0}<t_{1}<$ $\cdots<t_{n}=t$. In the present paper, with the conditioning functions $X_{t}$ and $Y_{t}$, we introduce two simple formulas for the conditional expectations over $C^{r}[0, t]$, an analogue of the $r$-dimensional Wiener space. We establish evaluation formulas for the analogues of the analytic Wiener and Feynman integrals for the function

$$
G(x)=\exp \left\{\int_{0}^{t} \theta(s, x(s)) d \eta(s)\right\} \psi(x(t)),
$$

where $\theta(s, \cdot)$ and $\psi$ are the Fourier-Stieltjes transforms of the complex Borel measures on $\mathbb{R}^{r}$. Using the simple formulas, we evaluate the analogues of the conditional analytic Wiener and Feynman integrals of the functional $G$.
\end{abstract}

\section{Introduction and an analogue of the $r$-dimensional Wiener space}

Let $C_{0}[0, t]$ be the space of real-valued continuous functions $x$ on $[0, t]$ with $x(0)=0$. It is well known that the space $C_{0}[0, t]$ is equipped with the Wiener measure which is a probability measure. On the space, Yeh introduced an inversion formula that a conditional expectation can be found by a Fouriertransform $([14,15,16])$. But Yeh's inversion formula is very complicated in its applications when the conditioning function is vector-valued. Let $\tau: 0=t_{0}<$ $t_{1}<\cdots<t_{n}=t$ be a partition of the interval $[0, t]$. In [12], Park and Skoug derived a simple formula for conditional Wiener integrals on $C_{0}[0, t]$ with the

Received November 10, 2009; Revised May 15, 2010.

2010 Mathematics Subject Classification. Primary 28C20.

Key words and phrases. analogue of Wiener measure, conditional Feynman integral, conditional Wiener integral, simple formula for conditional Wiener integral, Wiener space, Wiener integration formula.

This work was supported by the Korea Research Foundation Grant funded by the Korean Government(KRF-2008-313-C00029). 
conditioning function $X_{\tau}: C_{0}[0, t] \rightarrow \mathbb{R}^{n}$ given by

$$
X_{\tau}(x)=\left(x\left(t_{1}\right), \ldots, x\left(t_{n}\right)\right) .
$$

This formula expresses the conditional Wiener integrals directly in terms of ordinary Wiener integrals.

In [2], the author and his co-authors introduced a simple formula for conditional Wiener integrals over $C_{0}(\mathbb{B})$, the space of the abstract Wiener space valued continuous functions which vanish at 0 . Using the formula, they established various formulas for the conditional Wiener and Feynman integrals of the functionals on $C_{0}(\mathbb{B})$ in a certain Banach algebra which corresponds to the Cameron and Storvick's Banach algebra $\mathcal{S}^{\prime \prime}([1])$. In the paper, they evaluated the conditional Wiener and Feynman integral for the functionals of the form

$$
\exp \left\{\int_{0}^{t} \theta(s, x(s)) d \eta(s)\right\} \psi(x(t))
$$

where $\theta(s, \cdot)$ and $\psi$ are the Fourier-Stieltjes transforms of the complex Borel measures on a real separable Hilbert space $\mathcal{H}$. Note that $\theta(s, \cdot)$ and $\psi$ are defined on $\mathbb{B}$, the abstract Wiener space $([11])$. In [3], the author derived a simple formula for the conditional Wiener integrals over the product space $C_{0}(\mathbb{B}) \times C_{0}(\mathbb{B})$ and evaluated analytic Feynman integrals and conditional analytic Feynman integrals of the functionals in Banach algebras which are equivalent to the space of complex Borel measures on $\mathcal{H}([10])$.

On the other hand, let $C[0, t]$ denote the space of the real-valued continuous functions on the interval $[0, t]$. Ryu and Im introduced a probability measure $w_{\varphi}$ on $(C[0, t], \mathcal{B}(C[0, t]))$, where $\mathcal{B}(C[0, t])$ denotes the Borel $\sigma$-algebra on $C[0, t]$ and $\varphi$ is a probability distribution on $(\mathbb{R}, \mathcal{B}(\mathbb{R}))([7,13])$. This measure space is a generalization of the Wiener space $C_{0}[0, t]$. In the Wiener space, every path $x$ starts at the origin, that is, $x(0)=0$. If one considers all paths $x$ starting at an (arbitrary) point $c$, i.e., $x(0)=c$, then certain theories on the space $C_{0}[0, t]$ cannot hold or some of them must be modified. Fortunately, in $[4,6]$, the author could derive two simple formulas for the conditional $w_{\varphi}$-integrals of the functions on $C[0, t]$ with the vector-valued conditioning functions $X_{n}: C[0, t] \rightarrow$ $\mathbb{R}^{n+1}$ and $X_{n-1}: C[0, t] \rightarrow \mathbb{R}^{n}$ given by $X_{n}(x)=\left(x\left(t_{0}\right), x\left(t_{1}\right), \ldots, x\left(t_{n}\right)\right)$ and $X_{n-1}(x)=\left(x\left(t_{0}\right), x\left(t_{1}\right), \ldots, x\left(t_{n-1}\right)\right)$. These formulas express the conditional $w_{\varphi}$-integrals directly in terms of the non-conditional $w_{\varphi}$-integrals.

Let $C^{r}[0, t]$ be the product space of $C[0, t]$ and define $X_{t}: C^{r}[0, t] \rightarrow \mathbb{R}^{(n+1) r}$ and $Y_{t}: C^{r}[0, t] \rightarrow \mathbb{R}^{n r}$ by $X_{t}(x)=\left(x\left(t_{0}\right), x\left(t_{1}\right), \ldots, x\left(t_{n-1}\right), x\left(t_{n}\right)\right)$ and $Y_{t}(x)=\left(x\left(t_{0}\right), x\left(t_{1}\right), \ldots, x\left(t_{n-1}\right)\right)$. In [5], using the simple formula for the conditional expectations of the functions on $C^{r}[0, t]$ given $X_{t}$, the author evaluated the conditional analytic Feynman integral $E^{a n f_{q}}\left[F_{t} \mid X_{t}\right]$ for the function given by

$$
F_{t}(x)=\exp \left\{\int_{0}^{t} \theta(s, x(s)) d s\right\} \text { for } x \in C^{r}[0, t]
$$


where $\theta(s, \cdot)$ are the Fourier-Stieltjes transforms of the complex Borel measures on $\mathbb{R}^{r}$, and provided an inversion formula for $E^{a n f_{q}}\left[F_{t} \mid X_{t}\right]$.

In the present paper, with the conditioning functions $X_{t}$ and $Y_{t}$, we introduce two simple formulas for the conditional expectations over $C^{r}[0, t]$, an analogue of the $r$-dimensional Wiener space. We establish evaluation formulas for the analogues of the analytic Wiener and Feynman integrals for the function

$$
G(x)=\exp \left\{\int_{0}^{t} \theta(s, x(s)) d \eta(s)\right\} \psi(x(t)) \text { for } x \in C^{r}[0, t],
$$

where $\psi$ is the Fourier-Stieltjes transform of the complex Borel measures on $\mathbb{R}^{r}$. Using the simple formulas, we evaluate the analogues of the conditional analytic Wiener and Feynman integrals of the functional $G$.

Throughout this paper, let $\mathbb{C}$ and $\mathbb{C}_{+}$denote the set of the complex numbers and the set of the complex numbers with positive real parts, respectively.

Now, we begin with introducing the probability measure $w_{\varphi}$ on $(C[0, t]$, $\mathcal{B}(C[0, t]))$.

For a positive real $t$, let $C=C[0, t]$ be the space of all real-valued continuous functions on the closed interval $[0, t]$ with the supremum norm. For $\vec{t}=\left(t_{0}, t_{1}, \ldots, t_{n}\right)$ with $0=t_{0}<t_{1}<\cdots<t_{n} \leq t$, let $J_{\vec{t}}: C[0, t] \rightarrow \mathbb{R}^{n+1}$ be the function given by

$$
J_{\vec{t}}(x)=\left(x\left(t_{0}\right), x\left(t_{1}\right), \ldots, x\left(t_{n}\right)\right) .
$$

For $B_{j}(j=0,1, \ldots, n)$ in $\mathcal{B}(\mathbb{R})$, the subset $J_{\vec{t}}^{-1}\left(\prod_{j=0}^{n} B_{j}\right)$ of $C[0, t]$ is called an interval and let $\mathcal{I}$ be the set of all such intervals. For a probability measure $\varphi$ on $(\mathbb{R}, \mathcal{B}(\mathbb{R}))$, let

$$
\begin{aligned}
m_{\varphi}\left(J_{\vec{t}}^{-1}\left(\prod_{j=0}^{n} B_{j}\right)\right)= & {\left[\prod_{j=1}^{n} \frac{1}{2 \pi\left(t_{j}-t_{j-1}\right)}\right]^{\frac{1}{2}} \int_{B_{0}} \int_{\prod_{j=1}^{n} B_{j}} } \\
& \exp \left\{-\frac{1}{2} \sum_{j=1}^{n} \frac{\left(u_{j}-u_{j-1}\right)^{2}}{t_{j}-t_{j-1}}\right\} d\left(u_{1}, \ldots, u_{n}\right) d \varphi\left(u_{0}\right) .
\end{aligned}
$$

$\mathcal{B}(C[0, t])$ coincides with the smallest $\sigma$-algebra generated by $\mathcal{I}$ and there exists a unique probability measure $w_{\varphi}$ on $(C[0, t], \mathcal{B}(C[0, t]))$ such that $w_{\varphi}(I)=$ $m_{\varphi}(I)$ for all $I$ in $\mathcal{I}$. This measure $w_{\varphi}$ is called an analogue of the Wiener measure associated with the probability measure $\varphi([7,13])$. Let $r$ be a positive integer and $C^{r}=C^{r}[0, t]$ be the product space of $C[0, t]$ with the product measure $w_{\varphi}^{r}$. Since $C[0, t]$ is a separable Banach space, we have $\mathcal{B}\left(C^{r}[0, t]\right)=$ $\prod_{j=1}^{r} \mathcal{B}(C[0, t])$. This probability measure space $\left(C^{r}[0, t], \mathcal{B}\left(C^{r}[0, t]\right), w_{\varphi}^{r}\right)$ is called an analogue of the $r$-dimensional Wiener space.

Lemma 1.1 ([7, Lemma 2.1]). If $f: \mathbb{R}^{n+1} \rightarrow \mathbb{C}$ is a Borel measurable function, then we have

$$
\int_{C} f\left(x\left(t_{0}\right), x\left(t_{1}\right), \ldots, x\left(t_{n}\right)\right) d w_{\varphi}(x)
$$




$$
\begin{aligned}
\stackrel{*}{=} & {\left[\prod_{j=1}^{n} \frac{1}{2 \pi\left(t_{j}-t_{j-1}\right)}\right]^{\frac{1}{2}} \int_{\mathbb{R}} \int_{\mathbb{R}^{n}} f\left(u_{0}, u_{1}, \ldots, u_{n}\right) } \\
& \times \exp \left\{-\frac{1}{2} \sum_{j=1}^{n} \frac{\left(u_{j}-u_{j-1}\right)^{2}}{t_{j}-t_{j-1}}\right\} d\left(u_{1}, \ldots, u_{n}\right) d \varphi\left(u_{0}\right),
\end{aligned}
$$

where $\stackrel{*}{=}$ means that if either side exists, then both sides exist and they are equal.

Definition. Let $F: C^{r}[0, t] \rightarrow \mathbb{C}$ be integrable and let $X$ be a random vector on $C^{r}[0, t]$ assuming that the value space of $X$ is a normed space with the Borel $\sigma$-algebra. Then, we have the conditional expectation $E[F \mid X]$ of $F$ given $X$ from a well known probability theory. Further, there exists a $P_{X}$-integrable complex-valued function $\psi$ on the value space of $X$ such that $E[F \mid X](x)=$ $(\psi \circ X)(x)$ for $w_{\varphi}^{r}$-a.e. $x \in C^{r}[0, t]$, where $P_{X}$ is the probability distribution of $X$. The function $\psi$ is called the conditional $w_{\varphi}^{r}$-integral of $F$ given $X$ and it is also denoted by $E[F \mid X]$.

\section{The simple formulas for conditional $w_{\varphi}^{r}$-integrals}

Let $0=t_{0}<t_{1}<\cdots<t_{n-1}<t_{n}=t$ be a partition of $[0, t]$. For any $x$ in $C[0, t]$, define the polygonal function $[x]$ on $[0, t]$ by

$$
[x](s)=x\left(t_{j-1}\right)+\frac{s-t_{j-1}}{t_{j}-t_{j-1}}\left(x\left(t_{j}\right)-x\left(t_{j-1}\right)\right), t_{j-1} \leq s \leq t_{j}, j=1, \ldots, n .
$$

Similarly, for $\vec{\xi}_{n}=\left(\xi_{0}, \xi_{1}, \ldots, \xi_{n}\right) \in \mathbb{R}^{n+1}$, we define the polygonal function $\left[\vec{\xi}_{n}\right]$ of $\vec{\xi}_{n}$ replacing $x\left(t_{j}\right)$ by $\xi_{j}$. Similar definitions are understood when $x \in C^{r}[0, t]$ and $\vec{\xi}_{n}=\left(\xi_{0}, \xi_{1}, \ldots, \xi_{n}\right) \in \mathbb{R}^{(n+1) r}$.

Now, we introduce a lemma which is useful in proving several theorems. The proof follows immediately from Corollary 2.5 in [4].

Lemma 2.1. The processes $\left\{x(s)-[x](s): t_{j-1} \leq s \leq t_{j}\right\}$ on $C^{r}[0, t]$, where $j=1, \ldots, n$, are stochastically independent.

Let $\tau_{j}: 0=t_{j, 0}<t_{j, 1}<\cdots<t_{j, n_{j}}=t$ be a partition of $[0, t]$ and define $X_{\tau_{j}}: C[0, t] \rightarrow \mathbb{R}^{n_{j}+1}$ by $X_{\tau_{j}}(x)=\left(x\left(t_{j, 0}\right), x\left(t_{j, 1}\right), \ldots, x\left(t_{j, n_{j}}\right)\right)$ for $j=1, \ldots, r$. Further define $Y_{\tau_{j}}: C[0, t] \rightarrow \mathbb{R}^{n_{j}}$ by $Y_{\tau_{j}}(x)=\left(x\left(t_{j, 0}\right), x\left(t_{j, 1}\right), \ldots, x\left(t_{j, n_{j}-1}\right)\right)$.

In the following theorems, we introduce two simple formulas for the conditional $w_{\varphi}^{r}$-integrals on $C^{r}[0, t]$. Their proofs follow immediately from Theorem 2.9 in [4] and Theorem 2.5 in [6].

Theorem 2.2. Let $F: C^{r}[0, t] \rightarrow \mathbb{C}$ be integrable and $X_{t}: C^{r}[0, t] \rightarrow \prod_{j=1}^{r}$ $\mathbb{R}^{n_{j}+1}$ be given by

$$
X_{t}(x)=\left(X_{\tau_{1}}\left(x_{1}\right), \ldots, X_{\tau_{r}}\left(x_{r}\right)\right)
$$


for $x=\left(x_{1}, \ldots, x_{r}\right) \in C^{r}[0, t]$. Then we have for $P_{X_{t}}$-a.e. $\vec{\xi}=\left(\vec{\xi}_{1, n_{1}}, \ldots, \vec{\xi}_{r, n_{r}}\right)$ $\in \prod_{j=1}^{r} \mathbb{R}^{n_{j}+1}$

(2) $E\left[F \mid X_{t}\right](\vec{\xi})=E\left[F\left(x_{1}-\left[x_{1}\right]+\left[\vec{\xi}_{1, n_{1}}\right], \ldots, x_{r}-\left[x_{r}\right]+\left[\vec{\xi}_{r, n_{r}}\right]\right)\right]$,

where $P_{X_{t}}$ is the probability distribution of $X_{t}$ on $\left(\prod_{j=1}^{r} \mathbb{R}^{n_{j}+1}, \mathcal{B}\left(\prod_{j=1}^{r} \mathbb{R}^{n_{j}+1}\right)\right)$, and both $\left[x_{j}\right]$ and $\left[\vec{\xi}_{j, n_{j}}\right]$ are the polygonal functions with respect to the partition $\tau_{j}$ for $j=1, \ldots, r$.

Theorem 2.3. Let $F: C^{r}[0, t] \rightarrow \mathbb{C}$ be integrable and $Y_{t}: C^{r}[0, t] \rightarrow \prod_{j=1}^{r} \mathbb{R}^{n_{j}}$ be given by

$$
Y_{t}(x)=\left(Y_{\tau_{1}}\left(x_{1}\right), \ldots, Y_{\tau_{r}}\left(x_{r}\right)\right)
$$

for $x=\left(x_{1}, \ldots, x_{r}\right) \in C^{r}[0, t]$. Then we have for $P_{Y_{t}}$-a.e. $\vec{\xi}_{-1}=\left(\vec{\xi}_{1, n_{1}-1}, \ldots\right.$, $\left.\vec{\xi}_{r, n_{r}-1}\right) \in \prod_{j=1}^{r} \mathbb{R}^{n_{j}}$

$$
\begin{aligned}
& E\left[F \mid Y_{t}\right]\left(\vec{\xi}_{-1}\right) \\
= & {\left[\prod_{j=1}^{r} \frac{1}{2 \pi\left(t-t_{n_{j}-1}\right)}\right]^{\frac{1}{2}} \int_{\mathbb{R}^{r}} E\left[F \left(x_{1}-\left[x_{1}\right]+\left[\left(\vec{\xi}_{1, n_{1}-1}, \xi_{1, n_{1}}\right)\right], \ldots,\right.\right.} \\
& \left.\left.x_{r}-\left[x_{r}\right]+\left[\left(\vec{\xi}_{r, n_{r}-1}, \xi_{r, n_{r}}\right)\right]\right)\right] \exp \left\{-\frac{1}{2} \sum_{j=1}^{r} \frac{\left(\xi_{j, n_{j}}-\xi_{j, n_{j}-1}\right)^{2}}{t-t_{n_{j}-1}}\right\} \\
& d\left(\xi_{1, n_{1}}, \ldots, \xi_{r, n_{r}}\right),
\end{aligned}
$$

where $P_{Y_{t}}$ is the probability distribution of $Y_{t}$ on $\left(\prod_{j=1}^{r} \mathbb{R}^{n_{j}}, \mathcal{B}\left(\prod_{j=1}^{r} \mathbb{R}^{n_{j}}\right)\right)$ and $\vec{\xi}_{j, n_{j}-1}=\left(\xi_{j, 0}, \xi_{j, 1}, \ldots, \xi_{j, n_{j}-1}\right) \in \mathbb{R}^{n_{j}}$ for $j=1, \ldots, r$.

For a function $F: C^{r}[0, t] \rightarrow \mathbb{C}$ and $\lambda>0$, let $F^{\lambda}(x)=F\left(\lambda^{-\frac{1}{2}} x\right), X_{t}^{\lambda}(x)=$ $X_{t}\left(\lambda^{-\frac{1}{2}} x\right)$ and $Y_{t}^{\lambda}(x)=Y_{t}\left(\lambda^{-\frac{1}{2}} x\right)$, where $X_{t}$ and $Y_{t}$ are given by (1) and (3), respectively. Suppose that $E\left[F^{\lambda}\right]$ exists for each $\lambda>0$. By the definition of the conditional $w_{\varphi}^{r}$-integral and (2), we have

$$
E\left[F^{\lambda} \mid X_{t}^{\lambda}\right](\vec{\xi})=E\left[F\left(\lambda^{-\frac{1}{2}}\left(x_{1}-\left[x_{1}\right]\right)+\left[\vec{\xi}_{1, n_{1}}\right], \ldots, \lambda^{-\frac{1}{2}}\left(x_{r}-\left[x_{r}\right]\right)+\left[\vec{\xi}_{r, n_{r}}\right]\right)\right]
$$

for $P_{X_{t}^{\lambda}}$-a.e. $\vec{\xi}=\left(\vec{\xi}_{1, n_{1}}, \ldots, \vec{\xi}_{r, n_{r}}\right) \in \prod_{j=1}^{r} \mathbb{R}^{n_{j}+1}$, where $P_{X_{t}^{\lambda}}$ is the probability distribution of $X_{t}^{\lambda}$ on the Borel class of $\prod_{j=1}^{r} \mathbb{R}^{n_{j}+1}$. Throughout this paper, let

$$
I_{F}^{\lambda}(\vec{\xi})=E\left[F\left(\lambda^{-\frac{1}{2}}\left(x_{1}-\left[x_{1}\right]\right)+\left[\vec{\xi}_{1, n_{1}}\right], \ldots, \lambda^{-\frac{1}{2}}\left(x_{r}-\left[x_{r}\right]\right)+\left[\vec{\xi}_{r, n_{r}}\right]\right)\right]
$$

unless otherwise specified. Moreover, under the notations used in Theorem 2.3, we have by (4)

$$
\begin{aligned}
& E\left[F^{\lambda} \mid Y_{t}^{\lambda}\right]\left(\vec{\xi}_{-1}\right) \\
= & {\left[\prod_{j=1}^{r} \frac{\lambda}{2 \pi\left(t-t_{n_{j}-1}\right)}\right]^{\frac{1}{2}} \int_{\mathbb{R}^{r}} E\left[F \left(\lambda^{-\frac{1}{2}}\left(x_{1}-\left[x_{1}\right]\right)+\right.\right.}
\end{aligned}
$$




$$
\begin{aligned}
& \left.\left.\left[\left(\vec{\xi}_{1, n_{1}-1}, \xi_{1, n_{1}}\right)\right], \ldots, \lambda^{-\frac{1}{2}}\left(x_{r}-\left[x_{r}\right]\right)+\left[\left(\vec{\xi}_{r, n_{r}-1}, \xi_{r, n_{r}}\right)\right]\right)\right] \\
& \times \exp \left\{-\frac{\lambda}{2} \sum_{j=1}^{r} \frac{\left(\xi_{j, n_{j}}-\xi_{j, n_{j}-1}\right)^{2}}{t-t_{n_{j}-1}}\right\} d\left(\xi_{1, n_{1}}, \ldots, \xi_{r, n_{r}}\right)
\end{aligned}
$$

for $P_{Y_{t}^{\lambda}}$-a.e. $\vec{\xi}_{-1} \in \prod_{j=1}^{r} \mathbb{R}^{n_{j}}$, where $P_{Y_{t}^{\lambda}}$ is the probability distribution of $Y_{t}^{\lambda}$ on the Borel class of $\prod_{j=1}^{r} \mathbb{R}^{n_{j}}$. From now on, let $K_{F}^{\lambda}\left(\vec{\xi}_{-1}\right)$ be given by (5).

If $I_{F}^{\lambda}(\vec{\xi})$ has the analytic extension $J_{\lambda}^{*}(F)(\vec{\xi})$ on $\mathbb{C}_{+}$as a function of $\lambda$, then it is called the conditional analytic Wiener $w_{\varphi}^{r}$-integral of $F$ given $X_{t}$ with the parameter $\lambda$ and denoted by

$$
E^{a n w_{\lambda}}\left[F \mid X_{t}\right](\vec{\xi})=J_{\lambda}^{*}(F)(\vec{\xi})
$$

for $\vec{\xi} \in \prod_{j=1}^{r} \mathbb{R}^{n_{j}+1}$. Moreover, if for a nonzero real $q, E^{a n w_{\lambda}}\left[F \mid X_{t}\right](\vec{\xi})$ has the limit as $\lambda$ approaches to $-i q$ through $\mathbb{C}_{+}$, then it is called the conditional analytic Feynman $w_{\varphi}^{r}$-integral of $F$ given $X_{t}$ with the parameter $q$ and denoted by

$$
E^{a n f_{q}}\left[F \mid X_{t}\right](\vec{\xi})=\lim _{\lambda \rightarrow-i q} E^{a n w_{\lambda}}\left[F \mid X_{t}\right](\vec{\xi}) .
$$

Similar definitions are understood with $K_{F}^{\lambda}\left(\vec{\xi}_{-1}\right)$ if we replace $X_{t}$ by $Y_{t}$.

If $E\left[F^{\lambda}\right]$ itself has the analytic extension $J_{\lambda}^{*}(F)$ on $\mathbb{C}_{+}$, then we call $J_{\lambda}^{*}(F)$ the analytic Wiener $w_{\varphi}^{r}$-integral of $F$ over $C^{r}[0, t]$ with the parameter $\lambda$ and it is denoted by

$$
E^{a n w_{\lambda}}[F]=J_{\lambda}^{*}(F) .
$$

Further, if for a nonzero real $q, E^{a n w_{\lambda}}[F]$ has the limit as $\lambda$ approaches to $-i q$ through $\mathbb{C}_{+}$, then it is called the analytic Feynman $w_{\varphi}^{r}$-integral of $F$ over $C^{r}[0, t]$ with the parameter $q$ and denoted by

$$
E^{a n f_{q}}[F]=\lim _{\lambda \rightarrow-i q} E^{a n w_{\lambda}}[F] .
$$

\section{The analytic Wiener and Feynman $w_{\varphi}^{r}$-integrals}

Let $\eta$ be a complex valued Borel measure on $[0, t]$. Then $\eta=\mu+\nu$ can be decomposed uniquely into the sum of a continuous measure $\mu$ (with respect to the Lebesgue measure) and a discrete measure $\nu$. Further, let $\delta_{p_{j}}$ denote the Dirac measure with total mass 1 concentrated at $p_{j}$.

Let $\mathcal{M}\left(\mathbb{R}^{r}\right)$ be the class of all complex Borel measures on $\mathbb{R}^{r}$ and $\mathcal{G}^{*}$ be the set of all $\mathbb{C}$-valued functions $\theta$ on $[0, \infty) \times \mathbb{R}^{r}$ which have the form

$$
\theta(s, \vec{u})=\int_{\mathbb{R}^{r}} \exp \{i\langle\vec{u}, \vec{v}\rangle\} d \sigma_{s}(\vec{v}),
$$

where $\langle\cdot, \cdot\rangle$ denotes the dot product on $\mathbb{R}^{r}$ and $\left\{\sigma_{s}: s \in[0, \infty)\right\}$ is the family from $\mathcal{M}\left(\mathbb{R}^{r}\right)$ satisfying the following conditions;

(i) for each Borel subset $E$ of $\mathbb{R}^{r}, \sigma_{s}(E)$ is a Borel measurable function of $s$ on $[0, t]$, 
(ii) $\left\|\sigma_{s}\right\| \in L_{1}([0, t], \mathcal{B}([0, t]),|\eta|)$.

Theorem 3.1. Let $m$ and $k$ be two positive integers, and let $\eta=\mu+\sum_{j=1}^{m} w_{j} \delta_{p_{j}}$, where $0<p_{1}<\cdots<p_{m}<t$ and the $w_{j}$ s are in $\mathbb{C}$. Let $\theta \in \mathcal{G}^{*}$ be given by (6) and $F_{k}(x)=\left[\int_{0}^{t} \theta(s, x(s)) d \eta(s)\right]^{k}$ for $x \in C^{r}[0, t]$. Then for $\lambda>0$, we have

$$
E\left[F_{k}^{\lambda}\right]=\sum_{q_{0}+q_{1}+\cdots+q_{m}=k} \sum_{j_{0}+\cdots+j_{m}=q_{0}} H\left(k, \lambda, \overrightarrow{0}, q_{0}, q_{1}, \ldots, q_{m} ; j_{0}, \ldots, j_{m}\right),
$$

where for $\vec{v}_{m, j_{m}+1} \in \mathbb{R}^{r}$

$$
\begin{aligned}
& H\left(k, \lambda, \vec{v}_{m, j_{m}+1}, q_{0}, q_{1}, \ldots, q_{m} ; j_{0}, \ldots, j_{m}\right) \\
= & k !\left(\prod_{\alpha=1}^{m} \frac{w_{\alpha}^{q_{\alpha}}}{q_{\alpha} !}\right) \int_{\Delta_{q_{0} ; j_{0}, \ldots, j_{m}}} \int_{\mathbb{R}^{k r}} \exp \left\{-\frac{1}{2 \lambda} \sum_{\alpha=0}^{m} \sum_{\beta=1}^{j_{\alpha}+1}\left(s_{\alpha, \beta}-s_{\alpha, \beta-1}\right)\right. \\
& \left.\times\left\|\sum_{\gamma=\beta}^{j_{\alpha}+1} \vec{v}_{\alpha, \gamma}+\sum_{l=\alpha+1}^{m} \sum_{\gamma=1}^{j_{l}+1} \vec{v}_{l, \gamma}\right\|^{2}\right\} \int_{\mathbb{R}^{r}} \exp \left\{i \lambda^{-\frac{1}{2}}\left\langle\vec{\eta}, \sum_{\alpha=0}^{m} \sum_{\beta=1}^{j_{\alpha}+1} \vec{v}_{\alpha, \beta}\right\rangle\right\} d \varphi^{r}(\vec{\eta}) \\
& d\left(\prod_{\alpha=0}^{m} \prod_{\beta=1}^{j_{\alpha}} \sigma_{s_{\alpha, \beta}} \times \prod_{\alpha=1}^{m} \sigma_{p_{\alpha}}^{q_{\alpha}}\right)(\vec{v}, \vec{z}) d \mu^{q_{0}}(\vec{s})
\end{aligned}
$$

with $s_{0,0}=0, \vec{s}=\left(s_{0,1}, \ldots, s_{0, j_{0}}, s_{1,1}, \ldots, s_{1, j_{1}}, \ldots, s_{m, 1}, \ldots, s_{m, j_{m}}\right), s_{\alpha, 0}=$ $s_{\alpha-1, j_{\alpha-1}+1}=p_{\alpha}$ for $\alpha=1, \ldots, m, s_{m, j_{m}+1}=t, \Delta_{q_{0} ; j_{0}, \ldots, j_{m}}=\left\{\vec{s}: 0<s_{0,1}<\right.$ $\cdots<s_{0, j_{0}}<p_{1}<s_{1,1}<\cdots<s_{1, j_{1}}<p_{2}<\cdots<p_{m-1}<s_{m-1,1}<\cdots<$ $\left.s_{m-1, j_{m-1}}<p_{m}<s_{m, 1}<\cdots<s_{m, j_{m}}<t\right\}, \vec{v}=\left(\vec{v}_{0,1}, \ldots, \vec{v}_{0, j_{0}}, \vec{v}_{1,1}, \ldots, \vec{v}_{1, j_{1}}\right.$, $\left.\ldots, \vec{v}_{m, 1}, \ldots, \vec{v}_{m, j_{m}}\right)$ and $\vec{z}=\left(\vec{z}_{1,1}, \ldots, \vec{z}_{1, q_{1}}, \vec{z}_{2,1}, \ldots, \vec{z}_{2, q_{2}}, \ldots, \vec{z}_{m, 1}, \ldots, \vec{z}_{m, q_{m}}\right)$; $\vec{v}_{\alpha-1, j_{\alpha-1}+1}=\sum_{l=1}^{q_{\alpha}} \vec{z}_{\alpha, l}$ for $\alpha=1, \ldots, m$.

Proof. For $\lambda>0$, we have

$$
\begin{aligned}
E\left[F_{k}^{\lambda}\right]= & \sum_{q_{0}+q_{1}+\cdots+q_{m}=k} \frac{k !}{q_{0} ! q_{1} ! \cdots q_{m} !} \int_{C^{r}}\left[\int_{0}^{t} \theta\left(s, \lambda^{-\frac{1}{2}} x(s)\right) d \mu(s)\right]^{q_{0}} \\
& \times\left[\prod_{j=1}^{m} w_{j}^{q_{j}}\left[\theta\left(p_{j}, \lambda^{-\frac{1}{2}} x\left(p_{j}\right)\right)\right]^{q_{j}}\right] d w_{\varphi}^{r}(x)
\end{aligned}
$$

by the binomial expansion. Letting $\Delta_{q_{0}}=\left\{\left(s_{1}, \ldots, s_{q_{0}}\right): 0<s_{1}<\cdots<s_{q_{0}}<\right.$ $t\}$, we have by the simplex method ([8]) and the Fubini theorem

$$
\begin{aligned}
& E\left[F_{k}^{\lambda}\right] \\
= & k ! \sum_{q_{0}+q_{1}+\cdots+q_{m}=k}\left(\prod_{\alpha=1}^{m} \frac{w_{\alpha}^{q_{\alpha}}}{q_{\alpha} !}\right) \int_{C^{r}}\left[\sum_{j_{0}+\cdots+j_{m}=q_{0}} \int_{\Delta_{q_{0} ; j_{0}, \ldots, j_{m}}} \int_{\mathbb{R}_{q_{0} r}} \exp \{i\right. \\
& \left.\left.\times \lambda^{-\frac{1}{2}} \sum_{\alpha=0}^{m} \sum_{\beta=1}^{j_{\alpha}}\left\langle x\left(s_{\alpha, \beta}\right), \vec{v}_{\alpha, \beta}\right\rangle\right\} d\left(\prod_{\alpha=0}^{m} \prod_{\beta=1}^{j_{\alpha}} \sigma_{s_{\alpha, \beta}}\right)(\vec{v}) d \mu^{q_{0}}(\vec{s})\right]\left[\int_{\mathbb{R}^{\left(q_{1}+\cdots+q_{m}\right) r}}\right.
\end{aligned}
$$




$$
\left.\exp \left\{i \lambda^{-\frac{1}{2}} \sum_{\alpha=1}^{m} \sum_{l=1}^{q_{\alpha}}\left\langle x\left(s_{\alpha, 0}\right), \vec{z}_{\alpha, l}\right\rangle\right\} d\left(\prod_{\alpha=1}^{m} \sigma_{p_{\alpha}}^{q_{\alpha}}\right)(\vec{z})\right] d w_{\varphi}^{r}(x),
$$

where $\vec{v}=\left(\vec{v}_{0,1}, \ldots, \vec{v}_{0, j_{0}}, \vec{v}_{1,1}, \ldots, \vec{v}_{1, j_{1}}, \ldots, \vec{v}_{m, 1}, \ldots, \vec{v}_{m, j_{m}}\right)$ and $\vec{z}=\left(\vec{z}_{1,1}, \ldots\right.$, $\left.\vec{z}_{1, q_{1}}, \vec{z}_{2,1}, \ldots, \vec{z}_{2, q_{2}}, \ldots, \vec{z}_{m, 1}, \ldots, \vec{z}_{m, q_{m}}\right)$. Let $s_{0,0}=0, \vec{v}_{m, j_{m}+1}=\overrightarrow{0} \in \mathbb{R}^{r}$, $s_{m, j_{m}+1}=t$ and $\vec{v}_{\alpha-1, j_{\alpha-1}+1}=\sum_{l=1}^{q_{\alpha}} \vec{z}_{\alpha, l}$ for $\alpha=1, \ldots, m$. Then we have by an application of Lemma 1.1

$$
\begin{aligned}
& E\left[F_{k}^{\lambda}\right] \\
= & k ! \sum_{q_{0}+q_{1}+\cdots+q_{m}=k} \sum_{j_{0}+\cdots+j_{m}=q_{0}}\left(\prod_{\alpha=1}^{m} \frac{w_{\alpha}^{q_{\alpha}}}{q_{\alpha} !}\right) \int_{\Delta_{q_{0} ; j_{0}, \ldots, j_{m}}} \int_{\mathbb{R}^{k r}} \int_{C^{r}} \exp \left\{i \lambda^{-\frac{1}{2}}\right. \\
& \left.\times \sum_{\alpha=0}^{m} \sum_{\beta=1}^{j_{\alpha}+1}\left\langle x\left(s_{\alpha, \beta}\right), \vec{v}_{\alpha, \beta}\right\rangle\right\} d w_{\varphi}^{r}(x) d\left(\prod_{\alpha=0}^{m} \prod_{\beta=1}^{j_{\alpha}} \sigma_{s_{\alpha, \beta}} \times \prod_{\alpha=1}^{m} \sigma_{p_{\alpha}}^{q_{\alpha}}\right)(\vec{v}, \vec{z}) d \mu^{q_{0}}(\vec{s}) \\
= & k ! \sum_{q_{0}+q_{1}+\cdots+q_{m}=k} \sum_{j_{0}+\cdots+j_{m}=q_{0}}\left(\prod_{\alpha=1}^{m} \frac{w_{\alpha}^{q_{\alpha}}}{q_{\alpha} !}\right) \int_{\Delta_{q_{0} ; j_{0}, \ldots, j_{m}}} \int_{\mathbb{R}^{k r}}\left[\prod_{\alpha=0}^{m} \prod_{\beta=1}^{j_{\alpha}+1}\right. \\
& \left.\frac{1}{2 \pi\left(s_{\alpha, \beta}-s_{\alpha, \beta-1}\right)}\right]_{\mathbb{R}^{r}}^{\frac{r}{2}} \int_{\mathbb{R}^{\left(q_{0}+m+1\right) r}} \exp \left\{i \lambda^{-\frac{1}{2}} \sum_{\alpha=0}^{m} \sum_{\beta=1}^{j_{\alpha}+1}\left\langle\vec{\eta}_{\alpha, \beta}, \vec{v}_{\alpha, \beta}\right\rangle\right\} \\
& \times \exp \left\{-\frac{1}{2} \sum_{\alpha=0}^{m} \sum_{\beta=1}^{j_{\alpha}+1} \frac{\left\|\vec{\eta}_{\alpha, \beta}-\vec{\eta}_{\alpha, \beta-1}\right\|^{2}}{s_{\alpha, \beta}-s_{\alpha, \beta-1}} d\left(\vec{\eta}_{0,1}, \ldots, \vec{\eta}_{0, j_{0}+1}, \vec{\eta}_{1,1}, \ldots, \vec{\eta}_{1, j_{1}+1},\right.\right. \\
& \left.\ldots, \vec{\eta}_{m, 1}, \ldots, \vec{\eta}_{m, j_{m}+1}\right) d \varphi^{r}\left(\vec{\eta}_{0,0}\right) d\left(\prod_{\alpha=0}^{m} \prod_{\beta=1}^{j_{\alpha}} \sigma_{s_{\alpha, \beta}} \times \prod_{\alpha=1}^{m} \sigma_{p_{\alpha}}^{q_{\alpha}}\right)(\vec{v}, \vec{z}) d \mu^{q_{0}}(\vec{s}),
\end{aligned}
$$

where $\vec{\eta}_{\alpha, 0}=\vec{\eta}_{\alpha-1, j_{\alpha-1}+1}$ for $\alpha=1, \ldots, m$. For $\alpha=0, \ldots, m ; \beta=1, \ldots, j_{\alpha}+1$, let $\vec{\zeta}_{\alpha, \beta}=\vec{\eta}_{\alpha, \beta}-\vec{\eta}_{\alpha, \beta-1}$. Then $\vec{\eta}_{\alpha, \beta}=\sum_{l=0}^{\alpha-1} \sum_{\gamma=1}^{j_{l}+1} \vec{\zeta}_{l, \gamma}+\sum_{\gamma=1}^{\beta} \vec{\zeta}_{\alpha, \gamma}+\vec{\eta}_{0,0}$ and hence we have by the change of variable theorem

$$
\begin{aligned}
& E\left[F_{k}^{\lambda}\right] \\
= & k ! \sum_{q_{0}+q_{1}+\cdots+q_{m}=k} \sum_{j_{0}+\cdots+j_{m}=q_{0}}\left(\prod_{\alpha=1}^{m} \frac{w_{\alpha}^{q_{\alpha}}}{q_{\alpha} !}\right) \int_{\Delta_{q_{0} ; j_{0}, \ldots, j_{m}}} \int_{\mathbb{R}^{k r}}\left[\prod _ { \alpha = 0 } ^ { m } \prod _ { \beta = 1 } ^ { \frac { r } { 2 } } \int _ { \mathbb { R } ^ { r } } \int _ { \mathbb { R } ^ { ( q _ { 0 } + m + 1 ) r } } \operatorname { e x p } \left\{i \lambda ^ { - \frac { 1 } { 2 } } \sum _ { \alpha = 0 } ^ { m } \sum _ { \beta = 1 } ^ { j _ { \alpha } + 1 } \left\langle\sum_{l=0}^{\alpha-1} \sum_{\gamma=1}^{j_{l}+1} \vec{\zeta}_{l, \gamma}\right.\right.\right. \\
& \left.\left.\left.\frac{1}{2 \pi\left(s_{\alpha, \beta}-s_{\alpha, \beta-1}\right)}\right]_{\gamma=1}^{\beta} \vec{\zeta}_{\alpha, \gamma}+\vec{\eta}_{0,0}, \vec{v}_{\alpha, \beta}\right\rangle-\frac{1}{2} \sum_{\alpha=0}^{m} \sum_{\beta=1}^{j_{\alpha}+1} \frac{\left\|\vec{\zeta}_{\alpha, \beta}\right\|^{2}}{s_{\alpha, \beta}-s_{\alpha, \beta-1}}\right\} d \vec{\zeta} d \varphi^{r}\left(\vec{\eta}_{0,0}\right) \\
& d\left(\prod_{\alpha=0}^{m} \prod_{\beta=1}^{j_{\alpha}} \sigma_{s_{\alpha, \beta}} \times \prod_{\alpha=1}^{m} \sigma_{p_{\alpha}}^{q_{\alpha}}\right)(\vec{v}, \vec{z}) d \mu^{q_{0}}(\vec{s})
\end{aligned}
$$




$$
\begin{aligned}
& =k ! \sum_{q_{0}+q_{1}+\cdots+q_{m}=k} \sum_{j_{0}+\cdots+j_{m}=q_{0}}\left(\prod_{\alpha=1}^{m} \frac{w_{\alpha}^{q_{\alpha}}}{q_{\alpha} !}\right) \int_{\Delta_{q_{0} ; j_{0}, \ldots, j_{m}}} \int_{\mathbb{R}^{k r}}\left[\prod_{\alpha=0}^{m} \prod_{\beta=1}^{j_{\alpha}+1}\right. \\
& \left.\frac{1}{2 \pi\left(s_{\alpha, \beta}-s_{\alpha, \beta-1}\right)}\right]^{\frac{r}{2}} \int_{\mathbb{R}^{r}} \exp \left\{i \lambda^{-\frac{1}{2}}\left\langle\vec{\eta}, \sum_{\alpha=0}^{m} \sum_{\beta=1}^{j_{\alpha}+1} \vec{v}_{\alpha, \beta}\right\rangle\right\} \int_{\mathbb{R}^{\left(q_{0}+m+1\right) r}} \\
& \exp \left\{i \lambda^{-\frac{1}{2}} \sum_{\alpha=0}^{m} \sum_{\beta=1}^{j_{\alpha}+1}\left\langle\vec{\zeta}_{\alpha, \beta}, \sum_{\gamma=\beta}^{j_{\alpha}+1} \vec{v}_{\alpha, \gamma}+\sum_{l=\alpha+1}^{m} \sum_{\gamma=1}^{j_{l}+1} \vec{v}_{l, \gamma}\right\rangle-\frac{1}{2} \sum_{\alpha=0}^{m} \sum_{\beta=1}^{j_{\alpha}+1}\right. \\
& \left.\frac{\left\|\vec{\zeta}_{\alpha, \beta}\right\|^{2}}{s_{\alpha, \beta}-s_{\alpha, \beta-1}}\right\} d \vec{\zeta} d \varphi^{r}(\vec{\eta}) d\left(\prod_{\alpha=0}^{m} \prod_{\beta=1}^{j_{\alpha}} \sigma_{s_{\alpha, \beta}} \times \prod_{\alpha=1}^{m} \sigma_{p_{\alpha}}^{q_{\alpha}}\right)(\vec{v}, \vec{z}) d \mu^{q_{0}}(\vec{s}) \\
& =\sum_{q_{0}+q_{1}+\cdots+q_{m}=k} \sum_{j_{0}+\cdots+j_{m}=q_{0}} H\left(k, \lambda, \overrightarrow{0}, q_{0}, q_{1}, \ldots, q_{m} ; j_{0}, \ldots, j_{m}\right),
\end{aligned}
$$

where $\vec{\zeta}=\left(\vec{\zeta}_{0,1}, \ldots, \vec{\zeta}_{0, j_{0}+1}, \vec{\zeta}_{1,1}, \ldots, \vec{\zeta}_{1, j_{1}+1}, \ldots, \vec{\zeta}_{m, 1}, \ldots, \vec{\zeta}_{m, j_{m}+1}\right)$ and the last equality follows from the well known integration formula

$$
\int_{\mathbb{R}} \exp \left\{-a u^{2}+i b u\right\} d u=\left(\frac{\pi}{a}\right)^{\frac{1}{2}} \exp \left\{-\frac{b^{2}}{4 a}\right\}
$$

for $a \in \mathbb{C}_{+}$and any real $b$.

Corollary 3.2. Under the assumptions given as in Theorem 3.1 with one exception $\eta=\mu$, that is, assuming that $\eta$ has no discrete part, we have

$$
\begin{aligned}
E\left[F_{k}^{\lambda}\right]= & k ! \int_{\Delta_{k}} \int_{\mathbb{R}^{k r}} \exp \left\{-\frac{1}{2 \lambda} \sum_{l=1}^{k}\left(s_{l}-s_{l-1}\right)\left\|\sum_{\gamma=l}^{k} \vec{v}_{\gamma}\right\|^{2}\right\} \int_{\mathbb{R}^{r}} \\
& \exp \left\{i \lambda^{-\frac{1}{2}}\left\langle\vec{\eta}, \sum_{l=1}^{k} \vec{v}_{l}\right\rangle\right\} d \varphi^{r}(\vec{\eta}) d\left(\prod_{l=1}^{k} \sigma_{s_{l}}\right)(\vec{v}) d \mu^{k}(\vec{s}),
\end{aligned}
$$

where $s_{0}=0, \vec{s}=\left(s_{1}, \ldots, s_{k}\right), \vec{v}=\left(\vec{v}_{1}, \ldots, \vec{v}_{k}\right)$ and $\Delta_{k}=\left\{\vec{s}: 0<s_{1}<\cdots<\right.$ $\left.s_{k}<t\right\}$.

Corollary 3.3. Under the assumptions given as in Theorem 3.1 with one exception $\eta=\sum_{j=1}^{m} w_{j} \delta_{p_{j}}$, that is, assuming that $\eta$ has no continuous part, we have

$$
\begin{aligned}
& E\left[F_{k}^{\lambda}\right] \\
= & k ! \sum_{q_{1}+\cdots+q_{m}=k}\left(\prod_{\alpha=1}^{m} \frac{w_{\alpha}^{q_{\alpha}}}{q_{\alpha} !}\right) \int_{\mathbb{R}^{k r}} \exp \left\{-\frac{1}{2 \lambda} \sum_{\alpha=1}^{m}\left(p_{\alpha}-p_{\alpha-1}\right)\left\|\sum_{\gamma=\alpha}^{m} \sum_{l=1}^{q_{\gamma}} \vec{z}_{\gamma, l}\right\|^{2}\right\} \\
& \times \int_{\mathbb{R}^{r}} \exp \left\{i \lambda^{-\frac{1}{2}}\left\langle\vec{\eta}, \sum_{\alpha=1}^{m} \sum_{l=1}^{q_{\alpha}} \vec{z}_{\alpha, l}\right\rangle\right\} d \varphi^{r}(\vec{\eta}) d\left(\prod_{\alpha=1}^{m} \sigma_{p_{\alpha}}^{q_{\alpha}}\right)(\vec{z}),
\end{aligned}
$$

where $p_{0}=0, \vec{z}=\left(\vec{z}_{1,1}, \ldots, \vec{z}_{1, q_{1}}, \vec{z}_{2,1}, \ldots, \vec{z}_{2, q_{2}}, \ldots, \vec{z}_{m, 1}, \ldots, \vec{z}_{m, q_{m}}\right)$. 
Theorem 3.4. Let $\varphi^{r}$ be normally distributed with mean vector $\overrightarrow{0}$ and variancecovariance matrix $\sigma^{2} I_{r}$, where $I_{r}$ is the $r$-dimensional identity matrix. Then, under the assumptions and notations given as in Theorem 3.1, $E^{a n w_{\lambda}}\left[F_{k}\right]$ exists for $\lambda \in \mathbb{C}_{+}$and it is given by

$$
E^{a n w_{\lambda}}\left[F_{k}\right]=\sum_{q_{0}+q_{1}+\cdots+q_{m}=k} \sum_{j_{0}+\cdots+j_{m}=q_{0}} T\left(k, \lambda, \sigma, \overrightarrow{0}, q_{0}, \ldots, q_{m} ; j_{0}, \ldots, j_{m}\right),
$$

where for $\vec{v}_{m, j_{m}+1} \in \mathbb{R}^{r}, T\left(k, \lambda, \sigma, \vec{v}_{m, j_{m}+1}, q_{0}, \ldots, q_{m} ; j_{0}, \ldots, j_{m}\right)$ is given by the expression of $H\left(k, \lambda, \vec{v}_{m, j_{m}+1}, q_{0}, q_{1}, \ldots, q_{m} ; j_{0}, \ldots, j_{m}\right)$ replacing

$$
\int_{\mathbb{R}^{r}} \exp \left\{i \lambda^{-\frac{1}{2}}\left\langle\vec{\eta}, \sum_{\alpha=0}^{m} \sum_{\beta=1}^{j_{\alpha}+1} \vec{v}_{\alpha, \beta}\right\rangle\right\} d \varphi^{r}(\vec{\eta})
$$

by

$$
\exp \left\{-\frac{\sigma^{2}}{2 \lambda}\left\|\sum_{\alpha=0}^{m} \sum_{\beta=1}^{j_{\alpha}+1} \vec{v}_{\alpha, \beta}\right\|^{2}\right\}
$$

Furthermore, for nonzero real $q, E^{a n f_{q}}\left[F_{k}\right]$ exists and it is given by the above equality replacing $\lambda$ by $-i q$.

Proof. Under the notations given as in Theorem 3.1, we have for $\lambda>0$

$$
\begin{aligned}
& \int_{\mathbb{R}^{r}} \exp \left\{i \lambda^{-\frac{1}{2}}\left\langle\vec{\eta}, \sum_{\alpha=0}^{m} \sum_{\beta=1}^{j_{\alpha}+1} \vec{v}_{\alpha, \beta}\right\rangle\right\} d \varphi^{r}(\vec{\eta}) \\
= & \left(\frac{1}{\sqrt{2 \pi} \sigma}\right)^{r} \int_{\mathbb{R}^{r}} \exp \left\{i \lambda^{-\frac{1}{2}}\left\langle\vec{\eta}, \sum_{\alpha=0}^{m} \sum_{\beta=1}^{j_{\alpha}+1} \vec{v}_{\alpha, \beta}\right\rangle-\frac{1}{2 \sigma^{2}}\|\vec{\eta}\|^{2}\right\} d \vec{\eta} \\
= & \exp \left\{-\frac{\sigma^{2}}{2 \lambda}\left\|\sum_{\alpha=0}^{m} \sum_{\beta=1}^{j_{\alpha}+1} \vec{v}_{\alpha, \beta}\right\|^{2}\right\},
\end{aligned}
$$

where the last equality follows from (7). The results now follow by Theorem 3.1, Morera's theorem and the dominated convergence theorem.

Theorem 3.5. Let the assumptions and notations be given as in Theorem 3.1 and let $F(x)=\exp \left\{\int_{0}^{t} \theta(s, x(s)) d \eta(s)\right\}$ for $x \in C^{r}[0, t]$. Then for $\lambda>0$, we have

$$
E\left[F^{\lambda}\right]=1+\sum_{k=1}^{\infty} \frac{1}{k !} E\left[F_{k}^{\lambda}\right]
$$

where $E\left[F_{k}^{\lambda}\right]$ is given as in Theorem 3.1. Furthermore, under the assumptions given as in Theorem 3.4, $E^{a n f_{q}}[F]$ is obtained by

$$
E^{a n f_{q}}[F]=1+\sum_{k=1}^{\infty} \frac{1}{k !} E^{a n f_{q}}\left[F_{k}\right]
$$

for nonzero real $q$, where $E^{a n f_{q}}\left[F_{k}\right]$ is given as in Theorem 3.4. 
Proof. By the Maclaurin series of the exponential function, we have for $\lambda>0$

$$
F^{\lambda}(x)=1+\sum_{k=1}^{\infty} \frac{1}{k !} F_{k}^{\lambda}(x)
$$

and

$$
\begin{aligned}
\sum_{k=1}^{\infty} \frac{1}{k !}\left|F_{k}^{\lambda}(x)\right| & =\sum_{k=1}^{\infty} \frac{1}{k !}\left|\int_{0}^{t} \theta\left(s, \lambda^{-\frac{1}{2}} x(s)\right) d \eta(s)\right|^{k} \\
& \leq \sum_{k=1}^{\infty} \frac{1}{k !}\left[\int_{0}^{t}\left\|\sigma_{s}\right\| d|\eta|(s)\right]^{k}<\exp \left\{\int_{0}^{t}\left\|\sigma_{s}\right\| d|\eta|(s)\right\}
\end{aligned}
$$

Thus the convergence of (8) is uniform with respect to both $\lambda$ and $x$ which shows the first equality of the theorem. Applying the similar process to the results of Theorem 3.4, we can show the second equality of the theorem by Morera's theorem and the dominated convergence theorem.

Theorem 3.6. Let the assumptions and notations be given as Theorem 3.1 and let $G_{k}(x)=F_{k}(x) \psi(x(t))$ for $x \in C^{r}[0, t]$, where $\psi(\vec{u})=\int_{\mathbb{R}^{r}} \exp \{i\langle\vec{u}, \vec{v}\rangle\} d \nu(\vec{v})$ for $\nu \in \mathcal{M}\left(\mathbb{R}^{r}\right)$. Let $G(x)=\exp \left\{\int_{0}^{t} \theta(s, x(s)) d \eta(s)\right\} \psi(x(t))$. Then for $\lambda>0$, we have

$$
E\left[G^{\lambda}\right]=\int_{\mathbb{R}^{r}} \int_{\mathbb{R}^{r}} \exp \left\{i \lambda^{-\frac{1}{2}}\langle\vec{\eta}, \vec{v}\rangle-\frac{t}{2 \lambda}\|\vec{v}\|^{2}\right\} d \varphi^{r}(\vec{\eta}) d \nu(\vec{v})+\sum_{k=1}^{\infty} \frac{1}{k !} E\left[G_{k}^{\lambda}\right]
$$

where

$$
\begin{aligned}
& E\left[G_{k}^{\lambda}\right]=\sum_{q_{0}+q_{1}+\cdots+q_{m}=k} \sum_{j_{0}+\cdots+j_{m}=q_{0}} \int_{\mathbb{R}^{r}} H(k, \lambda, \\
& \left.\vec{v}_{m, j_{m}+1}, q_{0}, q_{1}, \ldots, q_{m} ; j_{0}, \ldots, j_{m}\right) d \nu\left(\vec{v}_{m, j_{m}+1}\right) .
\end{aligned}
$$

Furthermore, under the assumptions and notations given as in Theorem 3.4, $E^{a n f_{q}}[G]$ can be obtained by

$$
E^{a n f_{q}}[G]=\int_{\mathbb{R}^{r}} \exp \left\{\frac{t+\sigma^{2}}{2 q i}\|\vec{v}\|^{2}\right\} d \nu(\vec{v})+\sum_{k=1}^{\infty} \frac{1}{k !} E^{a n f_{q}}\left[G_{k}\right]
$$

for nonzero real $q$, where

$$
\begin{gathered}
E^{a n f_{q}}\left[G_{k}\right]=\sum_{\substack{\left.q_{0}+q_{1}+\cdots+q_{m}=k \\
q_{0}, \ldots, q_{m} ; j_{0}, \ldots, j_{m}\right) d \nu\left(\vec{v}_{m, j_{m}+1}\right) .}} \int_{\mathbb{R}^{r}} T\left(k,-i q, \sigma, \vec{v}_{m, j_{m}+1},\right.
\end{gathered}
$$

Proof. Using similar method used in the proof of Theorem 3.1 with an aid of

(7) we have for $\lambda>0$

$E\left[G_{k}^{\lambda}\right]$ 


$$
\begin{aligned}
& =k ! \sum_{q_{0}+q_{1}+\cdots+q_{m}=k}\left(\prod_{\alpha=1}^{m} \frac{w_{\alpha}^{q_{\alpha}}}{q_{\alpha} !}\right) \int_{C^{r}}\left[\sum_{j_{0}+\cdots+j_{m}=q_{0}} \int_{\Delta_{q_{0} ; j_{0}, \ldots, j_{m}}} \int_{\mathbb{R}^{q_{0} r}} \exp \{i\right. \\
& \left.\left.\times \lambda^{-\frac{1}{2}} \sum_{\alpha=0}^{m} \sum_{\beta=1}^{j_{\alpha}}\left\langle x\left(s_{\alpha, \beta}\right), \vec{v}_{\alpha, \beta}\right\rangle\right\} d\left(\prod_{\alpha=0}^{m} \prod_{\beta=1}^{j_{\alpha}} \sigma_{s_{\alpha, \beta}}\right)(\vec{v}) d \mu^{q_{0}}(\vec{s})\right]\left[\int_{\mathbb{R}^{\left(q_{1}+\cdots+q_{m}\right) r}}\right. \\
& \left.\exp \left\{i \lambda^{-\frac{1}{2}} \sum_{\alpha=1}^{m} \sum_{l=1}^{q_{\alpha}}\left\langle x\left(s_{\alpha, 0}\right), \vec{z}_{\alpha, l}\right\rangle\right\} d\left(\prod_{\alpha=1}^{m} \sigma_{p_{\alpha}}^{q_{\alpha}}\right)(\vec{z})\right]\left[\int _ { \mathbb { R } ^ { r } } \operatorname { e x p } \left\{i \lambda^{-\frac{1}{2}}\langle\right.\right. \\
& \left.\left.\left.x\left(s_{m, j_{m}+1}\right), \vec{v}_{m, j_{m}+1}\right\rangle\right\} d \nu\left(\vec{v}_{m, j_{m}+1}\right)\right] d w_{\varphi}^{r}(x) \\
& =k ! \sum_{q_{0}+q_{1}+\cdots+q_{m}=k}\left(\prod_{\alpha=1}^{m} \frac{w_{\alpha}^{q_{\alpha}}}{q_{\alpha} !}\right) \sum_{j_{0}+\cdots+j_{m}=q_{0}} \int_{\mathbb{R}^{r}} \int_{\Delta_{q_{0} ; j_{0}, \ldots, j_{m}}} \int_{\mathbb{R}^{k r}} \int_{C^{r}} \exp \{ \\
& \left.i \lambda^{-\frac{1}{2}} \sum_{\alpha=0}^{m} \sum_{\beta=1}^{j_{\alpha}+1}\left\langle x\left(s_{\alpha, \beta}\right), \vec{v}_{\alpha, \beta}\right\rangle\right\} d w_{\varphi}^{r}(x) d\left(\prod_{\alpha=0}^{m} \prod_{\beta=1}^{j_{\alpha}} \sigma_{s_{\alpha, \beta}} \times \prod_{\alpha=1}^{m} p_{\alpha}^{q_{\alpha}}\right)(\vec{v}, \vec{z}) \\
& d \mu^{q_{0}}(\vec{s}) d \nu\left(\vec{v}_{m, j_{m}+1}\right) \\
& =\sum_{q_{0}+q_{1}+\cdots+q_{m}=k} \sum_{j_{0}+\cdots+j_{m}=q_{0}} \int_{\mathbb{R}^{r}} H\left(k, \lambda, \vec{v}_{m, j_{m}+1}, q_{0}, q_{1}, \ldots, q_{m} ; j_{0}, \ldots, j_{m}\right) \\
& d \nu\left(\vec{v}_{m, j_{m}+1}\right) .
\end{aligned}
$$

Using the same process used in the proof of Theorem 3.5, we can prove the first part of the theorem. By the same method used in the proofs of Theorems 3.4 and 3.5, we have the second part of the theorem.

Corollary 3.7. Under the assumptions and notations given as in Theorem 3.6 with one exception $\varphi^{r}=\delta_{\overrightarrow{0}}$, the Dirac measure concentrated at $\overrightarrow{0} \in \mathbb{R}^{r}$, we have for a nonzero real $q$

$$
E^{a n f_{q}}[G]=\int_{\mathbb{R}^{r}} \exp \left\{\frac{t}{2 q i}\|\vec{v}\|^{2}\right\} d \nu(\vec{v})+\sum_{k=1}^{\infty} \frac{1}{k !} E^{a n f_{q}}\left[G_{k}\right]
$$

where

$$
\begin{aligned}
E^{a n f_{q}}\left[G_{k}\right]= & \sum_{\substack{q_{0}+q_{1}+\cdots+q_{m}=k \\
\vec{v}_{m, j_{m}+\cdots}, \cdots+j_{m}=q_{0}}} \int_{\mathbb{R}^{r}} T\left(k,-i q, 0, q_{m} ; j_{0}, \ldots, j_{m}\right) d \nu\left(\vec{v}_{m, j_{m}+1}\right)
\end{aligned}
$$

which is a main result of $[9]$.

Remark 3.8. - Under the conditions given as in Corollaries 3.2 and 3.3, we can obtain more simple expressions in Theorems 3.4, 3.5, 3.6 and Corollary 3.7.

- If $\eta=\mu+\sum_{j=1}^{m} w_{j} \delta_{p_{j}}$, where $0 \leq p_{1}<\cdots<p_{m} \leq t$, we can obtain all the results in the present section with minor modifications. 
- If $\eta=\mu+\sum_{j=1}^{\infty} w_{j} \delta_{p_{j}}$, then using the following version of the $\aleph_{0}$-nomial formula $([8, \mathrm{p} .41])$

$$
\left(\sum_{p=0}^{\infty} b_{p}\right)^{n}=\sum_{h=0}^{\infty} \sum_{q_{0}+q_{1}+\cdots+q_{h}=n, q_{h} \neq 0} \frac{n !}{q_{0} ! q_{1} ! \cdots q_{h} !} b_{0}^{q_{0}} b_{1}^{q_{1}} \cdots b_{h}^{q_{h}},
$$

we can show that for $\lambda>0, E\left[G^{\lambda}\right]$ exists in Theorem 3.6.

\section{The time-dependent conditional analytic Wiener and Feynman $w_{\varphi}^{r}$-integrals}

Let $\tau: 0=t_{0}<t_{1}<\cdots<t_{n}=t$ be a partition of $[0, t]$. From now on, we assume that $\tau_{1}=\cdots=\tau_{r}=\tau$, where the $\tau_{j}$ s are the partitions given as in Section 2. With these partitions let $[x]=\left(\left[x_{1}\right], \ldots,\left[x_{r}\right]\right)$ for $x=\left(x_{1}, \ldots, x_{r}\right) \in$ $C^{r}[0, t]$. In this case,

$$
[x](s)=x\left(t_{j-1}\right)+\frac{s-t_{j-1}}{t_{j}-t_{j-1}}\left(x\left(t_{j}\right)-x\left(t_{j-1}\right)\right)
$$

if $t_{j-1} \leq s \leq t_{j}$. Similarly, for $\vec{\xi}_{n+1}=\left(\vec{\xi}_{1, n}, \ldots, \vec{\xi}_{r, n}\right)=\left(\left(\xi_{1,0}, \ldots, \xi_{1, n}\right), \ldots\right.$, $\left.\left(\xi_{r, 0}, \ldots, \xi_{r, n}\right)\right) \in \mathbb{R}^{(n+1) r}$, let $\left[\vec{\xi}_{n+1}\right]=\left(\left[\vec{\xi}_{1, n}\right], \ldots,\left[\vec{\xi}_{r, n}\right]\right)$. If $t_{j-1} \leq s \leq t_{j}$, then we also have

$$
\left[\vec{\xi}_{n+1}\right](s)=\vec{\xi}_{j-1}+\frac{s-t_{j-1}}{t_{j}-t_{j-1}}\left(\vec{\xi}_{j}-\vec{\xi}_{j-1}\right)
$$

where $\vec{\xi}_{j}=\left(\xi_{1, j}, \ldots, \xi_{r, j}\right)$ for $j=0, \ldots, n$.

Our first theorem in this section is a time-dependent version of Theorem 3.1 above; its proof is similar and thus not included.

Theorem 4.1. Let the assumptions be given as in Theorem 3.1 and $X_{t}$ be given by (1). Further, let $\eta=\mu+\sum_{l=1}^{n} \sum_{j=1}^{r_{l}} w_{l, j} \delta_{p_{l, j}}$, where $w_{l, j} \in \mathbb{C}$ for all $(l, j)$ and $0=t_{0}<p_{1,1}<p_{1,2}<\cdots<p_{1, r_{1}}<t_{1}<p_{2,1}<\cdots<p_{2, r_{2}}<t_{2}<\cdots<$ $t_{n-1}<p_{n, 1}<\cdots<p_{n, r_{n}}<t_{n}=t$. Then for $\lambda \in \mathbb{C}_{+}$and $\vec{\xi}_{n+1} \in \mathbb{R}^{r(n+1)}$, $E^{a n w_{\lambda}}\left[F_{k} \mid X_{t}\right]\left(\vec{\xi}_{n+1}\right)$ is given by

$$
E^{a n w_{\lambda}}\left[F_{k} \mid X_{t}\right]\left(\vec{\xi}_{n+1}\right)=k ! \sum_{q_{1}+\cdots+q_{n}=k} \prod_{l=1}^{n} A\left(l, \lambda, \vec{\xi}_{n+1} ; q_{1}, \ldots, q_{n}\right),
$$

where

$$
\begin{aligned}
& A\left(l, \lambda, \vec{\xi}_{n+1} ; q_{1}, \ldots, q_{n}\right) \\
= & \sum_{m_{l, 0}+m_{l, 1}+\cdots+m_{l, r_{l}}=q_{l}}\left(\prod_{j=1}^{r_{l}} \frac{w_{l, j}^{m_{l, j}}}{m_{l, j} !}\right) \sum_{j_{0}+j_{1}+\cdots+j_{r_{l}}=m_{l, 0}} \int_{\Delta_{m_{l, 0} ; j_{0}, \ldots, j_{l}}} \int_{\mathbb{R}^{q_{l} r}} \\
& \exp \left\{i \sum_{u=0}^{r_{l}} \sum_{v=1}^{j_{u}+1}\left\langle\left[\vec{\xi}_{n+1}\right]\left(s_{l, u, v}\right), \vec{v}_{l, u, v}\right\rangle-\frac{1}{2 \lambda} \sum_{u=0}^{r_{l}} \sum_{v=1}^{j_{u}+1}\left(s_{l, u, v}-s_{l, u, v-1}\right) \| \sum_{\beta=u+1}^{r_{l}}\right.
\end{aligned}
$$




$$
\begin{aligned}
& \sum_{\gamma=1}^{j_{\beta}+1} \frac{t_{l}-s_{l, \beta, \gamma}}{t_{l}-t_{l-1}} \vec{v}_{l, \beta, \gamma}+\sum_{\gamma=v}^{j_{u}+1} \frac{t_{l}-s_{l, u, \gamma}}{t_{l}-t_{l-1}} \vec{v}_{l, u, \gamma}+\sum_{\gamma=1}^{v-1} \frac{t_{l-1}-s_{l, u, \gamma}}{t_{l}-t_{l-1}} \vec{v}_{l, u, \gamma}+\sum_{\beta=0}^{u-1} \\
& \left.\sum_{\gamma=1}^{j_{\beta}+1} \frac{t_{l-1}-s_{l, \beta, \gamma}}{t_{l}-t_{l-1}} \vec{v}_{l, \beta, \gamma} \|^{2}\right\} d\left(\prod_{u=0}^{r_{l}} \prod_{v=1}^{j_{u}} \sigma_{s_{l, u, v}} \times \prod_{u=1}^{r_{l}} \sigma_{p_{l, u}}^{m_{l, u}}\right)\left(\vec{v}_{l}, \vec{h}_{l}\right) d \mu^{m_{l, 0}\left(\vec{s}_{l}\right)}
\end{aligned}
$$

with $s_{l, 0,0}=t_{l-1}, s_{l, u, 0}=p_{l, u}=s_{l, u-1, j_{u-1}+1}$ for $u=1, \ldots, r_{l}, s_{l, r_{l}, j_{r_{l}}+1}=t_{l}$, $\vec{s}_{l}=\left(s_{l, 0,1}, \ldots, s_{l, 0, j_{0}}, \ldots, s_{l, r_{l}, 1}, \ldots, s_{l, r_{l}, j_{r_{l}}}\right), \Delta_{m_{l, 0} ; j_{0}, \ldots, j_{r_{l}}}=\left\{\begin{array}{l}\vec{s}_{l}: t_{l-1}< \\ s_{l, 0, s_{l}}<s_{l, r_{l}, 1}<\end{array}\right.$ $s_{l, 0,1}<\cdots<s_{l, 0, j_{0}}<p_{l, 1}<s_{l, 1,1}<\cdots<s_{l, 1, j_{1}}<p_{l, 2}<\cdots<p_{l, r_{l}}<s_{l, r_{l}, 1}<$ $\left.\ldots<s_{l, r_{l}, j_{r_{l}}}<t_{l}\right\}, \vec{v}_{l}=\left(\vec{v}_{l, 0,1}, \ldots, \vec{v}_{l, 0, j_{0}}, \vec{v}_{l, 1,1}, \ldots, \vec{v}_{l, 1, j_{1}}, \ldots, \vec{v}_{l, r_{l}, 1}, \ldots\right.$, $\left.\vec{v}_{l, r_{l}, j_{r_{l}}}\right), \vec{v}_{l, r_{l}, j_{r_{l}}+1}=\overrightarrow{0} \in \mathbb{R}^{r}$ and $\vec{h}_{l}=\left(\vec{h}_{l, 1,1}, \ldots, \vec{h}_{l, 1, m_{l, 1}}, \vec{h}_{l, 2,1}, \ldots, \vec{h}_{l, 2, m_{l, 2}}\right.$, $\left.\ldots, \vec{h}_{l, r_{l}, 1}, \ldots, \vec{h}_{l, r_{l}, m_{l, r_{l}}}\right) ; \vec{v}_{l, u-1, j_{u-1}+1}=\sum_{v=1}^{m_{l, u}} \vec{h}_{l, u, v}$ for $u=1, \ldots, r_{l}$. Furthermore, for nonzero real $q, E^{a n f_{q}}\left[F_{k} \mid X_{t}\right]\left(\vec{\xi}_{n+1}\right)$ is given by the right hand side of (10) replacing $\lambda$ by $-i q$.

By the same method used in the proof of Theorem 3.5, we can prove the following theorem.

Theorem 4.2. Let the assumptions and notations be given as in Theorem 4.1, and let $F$ be given as in Theorem 3.5. Then for nonzero real $q$ and $\vec{\xi}_{n+1} \in$ $\mathbb{R}^{(n+1) r}, E^{a n f_{q}}\left[F \mid X_{t}\right]\left(\vec{\xi}_{n+1}\right)$ exists and it is given by

$$
E^{a n f_{q}}\left[F \mid X_{t}\right]\left(\vec{\xi}_{n+1}\right)=1+\sum_{k=1}^{\infty} \frac{1}{k !} E^{a n f_{q}}\left[F_{k} \mid X_{t}\right]\left(\vec{\xi}_{n+1}\right),
$$

where $E^{a n f_{q}}\left[F_{k} \mid X_{t}\right]\left(\vec{\xi}_{n+1}\right)$ is given as in Theorem 4.1 .

Let $\psi(\vec{u})=\int_{\mathbb{R}^{r}} \exp \{i\langle\vec{u}, \vec{v}\rangle\} d \nu(\vec{v})$ for $\nu \in \mathcal{M}\left(\mathbb{R}^{r}\right)$. Then we have for $\vec{\xi}_{n+1}=$ $\left(\vec{\xi}_{0}, \vec{\xi}_{1}, \ldots, \vec{\xi}_{n}\right) \in \mathbb{R}^{r(n+1)}$ and $\lambda>0$

$$
\psi\left(\lambda^{-\frac{1}{2}}(x(t)-[x](t))+\left[\vec{\xi}_{n+1}\right](t)\right)=\psi\left(\vec{\xi}_{n}\right) .
$$

By (11) and Theorem 4.2, we have the following theorem.

Theorem 4.3. Let $G_{k}$ and $G$ be given as in Theorem 3.6. Then, under the assumptions and notations given as in Theorem 4.2, we have for nonzero real $q$ and $\vec{\xi}_{n+1}=\left(\vec{\xi}_{0}, \vec{\xi}_{1}, \ldots, \vec{\xi}_{n}\right) \in \mathbb{R}^{(n+1) r}$

$$
E^{a n f_{q}}\left[G_{k} \mid X_{t}\right]\left(\vec{\xi}_{n+1}\right)=\psi\left(\vec{\xi}_{n}\right) E^{a n f_{q}}\left[F_{k} \mid X_{t}\right]\left(\vec{\xi}_{n+1}\right)
$$

and

$$
\begin{aligned}
E^{a n f_{q}}\left[G \mid X_{t}\right]\left(\vec{\xi}_{n+1}\right) & =\psi\left(\vec{\xi}_{n}\right) E^{a n f_{q}}\left[F \mid X_{t}\right]\left(\vec{\xi}_{n+1}\right) \\
& =\psi\left(\vec{\xi}_{n}\right)+\sum_{k=1}^{\infty} \frac{1}{k !} E^{a n f_{q}}\left[G_{k} \mid X_{t}\right]\left(\vec{\xi}_{n+1}\right) .
\end{aligned}
$$


Remark 4.4. - If $F_{k}, G_{k}, F$ and $G$ are defined on the $r$-dimensional Wiener space, then we can obtain the same results in Theorems 4.1, 4.2 and 4.3 with $\vec{\xi}_{0}=\overrightarrow{0} \in \mathbb{R}^{r}$ in the expression of $\vec{\xi}_{n+1}$.

- If $\eta=\mu$ or $\eta=\sum_{l=1}^{n} \sum_{j=1}^{r_{l}} w_{l, j} \delta_{p_{l, j}}$, we can obtain more simple expressions in Theorems 4.1, 4.2 and 4.3 ([2, Corollaries 4.7 and 4.8]).

- If some of the $p_{l, j} \mathrm{~s}$ are in the set $\left\{t_{0}, t_{1}, \ldots, t_{n}\right\}$, we can obtain all the results in the present section with minor modifications.

- If $\eta=\mu+\sum_{l=1}^{n} \sum_{j=1}^{r_{l}} w_{l, j} \delta_{p_{l, j}}$ and some of the $r_{l}$ s are $\infty$, then, using (9), we can show that $E^{a n f_{q}}\left[G \mid X_{t}\right]$ exists in Theorem 4.3.

\section{The time-independent conditional analytic Wiener and Feynman $w_{\varphi}^{r}$-integrals}

In the present section we evaluate the conditional analytic Wiener and Feynman $w_{\varphi}^{r}$-integrals of $G$ given $Y_{t}$, where $G$ and $Y_{t}$ are given by Theorem 3.6 and (3), respectively. The following lemma follows easily using the change of variables theorem together with the equation (7).

Lemma 5.1. For $\lambda>0, \vec{v} \in \mathbb{R}^{r}$ and $\vec{\xi}=\left(\vec{\xi}_{0}, \vec{\xi}_{1}, \ldots, \vec{\xi}_{n-1}\right) \in \mathbb{R}^{n r}$ let

$$
\begin{aligned}
\Psi(n, \lambda, \vec{v}, \vec{\xi})= & {\left[\frac{\lambda}{2 \pi\left(t-t_{n-1}\right)}\right]^{\frac{r}{2}} \int_{\mathbb{R}^{r}} \exp \left\{i \sum _ { u = 0 } ^ { r _ { n } } \sum _ { v = 1 } ^ { j _ { u } + 1 } \left\langle\left[\left(\vec{\xi}, \vec{\xi}_{n}\right)\right]\left(s_{n, u, v}\right),\right.\right.} \\
& \left.\left.\vec{v}_{n, u, v}\right\rangle+i\left\langle\vec{\xi}_{n}, \vec{v}\right\rangle-\frac{\lambda}{2} \frac{\left\|\vec{\xi}_{n}-\vec{\xi}_{n-1}\right\|^{2}}{t-t_{n-1}}\right\} d \vec{\xi}_{n},
\end{aligned}
$$

where $\left(\vec{\xi}, \vec{\xi}_{n}\right)=\left(\vec{\xi}_{0}, \vec{\xi}_{1}, \ldots, \vec{\xi}_{n-1}, \vec{\xi}_{n}\right) \in \mathbb{R}^{(n+1) r}, t_{n-1} \leq s_{n, u, v} \leq t_{n}=t$ and $\vec{v}_{n, u, v} \in \mathbb{R}^{r}$ for $u=0,1, \ldots, r_{n} ; v=1,2, \ldots, j_{u}+1$. Then we have

$$
\begin{aligned}
\Psi(n, \lambda, \vec{v}, \vec{\xi})= & \exp \left\{i\left\langle\vec{\xi}_{n-1}, \vec{v}+\sum_{u=0}^{r_{n}} \sum_{v=1}^{j_{u}+1} \vec{v}_{n, u, v}\right\rangle\right. \\
& \left.-\frac{t-t_{n-1}}{2 \lambda}\left\|\vec{v}+\sum_{u=0}^{r_{n}} \sum_{v=1}^{j_{u}+1} \frac{s_{n, u, v}-t_{n-1}}{t-t_{n-1}} \vec{v}_{n, u, v}\right\|^{2}\right\} .
\end{aligned}
$$

Theorem 5.2. Let the assumptions and notations be given as in Theorem 4.1. Suppose that $Y_{t}$ is given by (3). Then for $\lambda \in \mathbb{C}_{+}$and $\vec{\xi}=\left(\vec{\xi}_{0}, \vec{\xi}_{1}, \ldots, \vec{\xi}_{n-1}\right) \in$ $\mathbb{R}^{n r}, E^{a n w_{\lambda}}\left[F_{k} \mid Y_{t}\right](\vec{\xi})$ is given by

$$
E^{a n w_{\lambda}}\left[F_{k} \mid Y_{t}\right](\vec{\xi})=k ! \sum_{q_{1}+\cdots+q_{n}=k}\left[\prod_{l=1}^{n-1} A\left(l, \lambda, \vec{\xi} ; q_{1}, \ldots, q_{n}\right)\right] B\left(n, \lambda, \overrightarrow{0}, \vec{\xi} ; q_{1}, \ldots, q_{n}\right),
$$

where for $\vec{v} \in \mathbb{R}^{r}, B\left(n, \lambda, \vec{v}, \vec{\xi} ; q_{1}, \ldots, q_{n}\right)$ is given by the expression of $A(n, \lambda, \vec{\xi}$; $\left.q_{1}, \ldots, q_{n}\right)$ replacing $\exp \left\{i \sum_{u=0}^{r_{n}} \sum_{v=1}^{j_{u}+1}\left\langle[\vec{\xi}]\left(s_{n, u, v}\right), \vec{v}_{n, u, v}\right\rangle\right\}$ by $\Psi(n, \lambda, \vec{v}, \vec{\xi})$ which is given by (12). Furthermore, for a nonzero real $q, E^{a n f_{q}}\left[F_{k} \mid Y_{t}\right](\vec{\xi})$ is given by the above expression replacing $\lambda$ by $-i q$. 
Proof. For $\vec{\xi}=\left(\vec{\xi}_{0}, \vec{\xi}_{1}, \ldots, \vec{\xi}_{n-1}\right) \in \mathbb{R}^{n r}$, let $\left(\vec{\xi}, \vec{\xi}_{n}\right)=\left(\vec{\xi}_{0}, \vec{\xi}_{1}, \ldots, \vec{\xi}_{n-1}, \vec{\xi}_{n}\right) \in$ $\mathbb{R}^{(n+1) r}$, where $\vec{\xi}_{n} \in \mathbb{R}^{r}$. Then for $\lambda>0$ we have by Theorem 4.1

$$
\begin{aligned}
K_{F_{k}}^{\lambda}(\vec{\xi})= & {\left[\frac{\lambda}{2 \pi\left(t-t_{n-1}\right)}\right]^{\frac{r}{2}} \int_{\mathbb{R}^{r}} I_{F_{k}}^{\lambda}\left(\vec{\xi}, \vec{\xi}_{n}\right) \exp \left\{-\frac{\lambda\left\|\vec{\xi}_{n}-\vec{\xi}_{n-1}\right\|^{2}}{2\left(t-t_{n-1}\right)}\right\} d \vec{\xi}_{n} } \\
= & k !\left[\frac{\lambda}{2 \pi\left(t-t_{n-1}\right)}\right]^{\frac{r}{2}} \int_{\mathbb{R}^{r}}\left[\sum_{q_{1}+\cdots+q_{n}=k} \prod_{l=1}^{n} A\left(l, \lambda,\left(\vec{\xi}, \vec{\xi}_{n}\right) ; q_{1}, \ldots, q_{n}\right)\right] \\
& \times \exp \left\{-\frac{\lambda\left\|\vec{\xi}_{n}-\vec{\xi}_{n-1}\right\|^{2}}{2\left(t-t_{n-1}\right)}\right\} d \vec{\xi}_{n} \\
= & k ! \sum_{q_{1}+\cdots+q_{n}=k}\left[\prod_{l=1}^{n-1} A\left(l, \lambda, \vec{\xi} ; q_{1}, \ldots, q_{n}\right)\right]\left[\frac{\lambda}{2 \pi\left(t-t_{n-1}\right)}\right]^{\frac{r}{2}} \\
& \times \int_{\mathbb{R}^{r}} A\left(n, \lambda,\left(\vec{\xi}, \vec{\xi}_{n}\right) ; q_{1}, \ldots, q_{n}\right) \exp \left\{-\frac{\lambda\left\|\vec{\xi}_{n}-\vec{\xi}_{n-1}\right\|^{2}}{2\left(t-t_{n-1}\right)}\right\} d \vec{\xi}_{n}
\end{aligned}
$$

with the convention that $\left[\left(\vec{\xi}, \vec{\xi}_{n}\right)\right]\left(s_{l, u, v}\right)=[\vec{\xi}]\left(s_{l, u, v}\right)$ if $0 \leq s_{l, u, v} \leq t_{n-1}$. Now by Theorem 4.1 and Lemma 5.1 we have

$$
\begin{aligned}
& {\left[\frac{\lambda}{2 \pi\left(t-t_{n-1}\right)}\right]^{\frac{r}{2}} \int_{\mathbb{R}^{r}} A\left(n, \lambda,\left(\vec{\xi}, \vec{\xi}_{n}\right) ; q_{1}, \ldots, q_{n}\right) \exp \left\{-\frac{\lambda\left\|\vec{\xi}_{n}-\vec{\xi}_{n-1}\right\|^{2}}{2\left(t-t_{n-1}\right)}\right\} d \vec{\xi}_{n} } \\
= & B\left(n, \lambda, \overrightarrow{0}, \vec{\xi} ; q_{1}, \ldots, q_{n}\right) .
\end{aligned}
$$

The theorem now follows from Morera's theorem and the dominated convergence theorem.

By the same method used in the proof of Theorem 3.5, we can prove the following theorem.

Theorem 5.3. Let the assumptions and notations be given as in Theorem 5.2 and let $F(x)=\exp \left\{\int_{0}^{t} \theta(s, x(s)) d \eta(s)\right\}$ for $x \in C^{r}[0, t]$. Then for nonzero real $q$ and $\vec{\xi} \in \mathbb{R}^{n r}, E^{a n f_{q}}\left[F \mid Y_{t}\right](\vec{\xi})$ exists and it is given by

$$
E^{a n f_{q}}\left[F \mid Y_{t}\right](\vec{\xi})=1+\sum_{k=1}^{\infty} \frac{1}{k !} E^{a n f_{q}}\left[F_{k} \mid Y_{t}\right](\vec{\xi}),
$$

where $E^{a n f_{q}}\left[F_{k} \mid Y_{t}\right](\vec{\xi})$ is given as in Theorem 5.2.

Theorem 5.4. Under the assumptions and notations given as in Theorems 4.3, 5.2 and 5.3, we have for nonzero real $q$ and $\vec{\xi}=\left(\vec{\xi}_{0}, \vec{\xi}_{1}, \ldots, \vec{\xi}_{n-1}\right) \in \mathbb{R}^{n r}$

$$
\begin{aligned}
E^{a n f_{q}}\left[G_{k} \mid Y_{t}\right](\vec{\xi})= & k ! \sum_{q_{1}+\cdots+q_{n}=k}\left[\prod_{l=1}^{n-1} A\left(l,-i q, \vec{\xi} ; q_{1}, \ldots, q_{n}\right)\right] \\
& \times \int_{\mathbb{R}^{r}} B\left(n,-i q, \vec{v}, \vec{\xi} ; q_{1}, \ldots, q_{n}\right) d \nu(\vec{v})
\end{aligned}
$$


and

$$
\begin{aligned}
E^{a n f_{q}}\left[G \mid Y_{t}\right](\vec{\xi})= & \int_{\mathbb{R}^{r}} \exp \left\{i\left\langle\vec{\xi}_{n-1}, \vec{v}\right\rangle+\frac{t-t_{n-1}}{2 q i}\|\vec{v}\|^{2}\right\} d \nu(\vec{v}) \\
& +\sum_{k=1}^{\infty} \frac{1}{k !} E^{a n f_{q}\left[G_{k} \mid Y_{t}\right](\vec{\xi})}
\end{aligned}
$$

Proof. For $\vec{\xi}=\left(\vec{\xi}_{0}, \vec{\xi}_{1}, \ldots, \vec{\xi}_{n-1}\right) \in \mathbb{R}^{n r}$, let $\left(\vec{\xi}, \vec{\xi}_{n}\right)=\left(\vec{\xi}_{0}, \vec{\xi}_{1}, \ldots, \vec{\xi}_{n-1}, \vec{\xi}_{n}\right) \in$ $\mathbb{R}^{(n+1) r}$, where $\vec{\xi}_{n} \in \mathbb{R}^{r}$. Then for $\lambda>0$ we have by Theorem 4.3

$$
\begin{aligned}
& K_{G_{k}}^{\lambda}(\vec{\xi}) \\
= & {\left[\frac{\lambda}{2 \pi\left(t-t_{n-1}\right)}\right]^{\frac{r}{2}} \int_{\mathbb{R}^{r}} I_{G_{k}}^{\lambda}\left(\vec{\xi}, \vec{\xi}_{n}\right) \exp \left\{-\frac{\lambda\left\|\vec{\xi}_{n}-\vec{\xi}_{n-1}\right\|^{2}}{2\left(t-t_{n-1}\right)}\right\} d \vec{\xi}_{n} } \\
= & k !\left[\frac{\lambda}{2 \pi\left(t-t_{n-1}\right)}\right]^{\frac{r}{2}} \int_{\mathbb{R}^{r}}\left[\sum_{q_{1}+\cdots+q_{n}=k} \prod_{l=1}^{n} A\left(l, \lambda,\left(\vec{\xi}, \vec{\xi}_{n}\right) ; q_{1}, \ldots, q_{n}\right)\right] \\
& \times \psi\left(\vec{\xi}_{n}\right) \exp \left\{-\frac{\lambda\left\|\vec{\xi}_{n}-\vec{\xi}_{n-1}\right\|^{2}}{2\left(t-t_{n-1}\right)}\right\} d \vec{\xi}_{n} \\
= & k ! \sum_{q_{1}+\cdots+q_{n}=k}\left[\prod_{l=1}^{n-1} A\left(l, \lambda, \vec{\xi} ; q_{1}, \ldots, q_{n}\right)\right]\left[\frac{\lambda}{2 \pi\left(t-t_{n-1}\right)}\right]^{\frac{r}{2}} \int_{\mathbb{R}^{r}} \int_{\mathbb{R}^{r}} \\
& A\left(n, \lambda,\left(\vec{\xi}, \vec{\xi}_{n}\right) ; q_{1}, \ldots, q_{n}\right) \exp \left\{i\left\langle\vec{\xi}_{n}, \vec{v}\right\rangle-\frac{\lambda\left\|\vec{\xi}_{n}-\vec{\xi}_{n-1}\right\|^{2}}{2\left(t-t_{n-1}\right)}\right\} d \vec{\xi}_{n} d \nu(\vec{v})
\end{aligned}
$$

with the convention that $\left[\left(\vec{\xi}, \vec{\xi}_{n}\right)\right]\left(s_{l, u, v}\right)=[\vec{\xi}]\left(s_{l, u, v}\right)$ if $0 \leq s_{l, u, v} \leq t_{n-1}$. Now by Theorem 4.1 and Lemma 5.1 we have

$$
\begin{aligned}
& {\left[\frac{\lambda}{2 \pi\left(t-t_{n-1}\right)}\right]^{\frac{r}{2}} \int_{\mathbb{R}^{r}} A\left(n, \lambda,\left(\vec{\xi}, \vec{\xi}_{n}\right) ; q_{1}, \ldots, q_{n}\right) } \\
& \times \exp \left\{i\left\langle\vec{\xi}_{n}, \vec{v}\right\rangle-\frac{\lambda\left\|\vec{\xi}_{n}-\vec{\xi}_{n-1}\right\|^{2}}{2\left(t-t_{n-1}\right)}\right\} d \vec{\xi}_{n} \\
= & B\left(n, \lambda, \vec{v}, \vec{\xi} ; q_{1}, \ldots, q_{n}\right) .
\end{aligned}
$$

The theorem now follows from Morera's theorem and the dominated convergence theorem.

Remark 5.5. - If $F_{k}, G_{k}, F$ and $G$ are defined on the $r$-dimensional Wiener space, then we can obtain the same results in Theorems 5.2, 5.3 and 5.4 with $\vec{\xi}_{0}=\overrightarrow{0} \in \mathbb{R}^{r}$ in the expression of $\vec{\xi}$.

- If $\eta=\mu$ or $\eta=\sum_{l=1}^{n} \sum_{j=1}^{r_{l}} w_{l, j} \delta_{p_{l, j}}$, we can obtain more simple expressions in Theorems 5.2, 5.3 and 5.4.

- If some of the $p_{l, j} \mathrm{~s}$ are in the set $\left\{t_{0}, t_{1}, \ldots, t_{n}\right\}$, we can obtain all the results in the present section with minor modifications. 
- If $\eta=\mu+\sum_{l=1}^{n} \sum_{j=1}^{r_{l}} w_{l, j} \delta_{p_{l, j}}$ and some of the $r_{l}$ s are $\infty$, then, using (9), we can show that $E^{a n f_{q}}\left[G \mid Y_{t}\right]$ exists in Theorem 5.4.

\section{References}

[1] R. H. Cameron and D. A. Storvick, Some Banach algebras of analytic Feynman integrable functionals, Analytic functions, Kozubnik 1979 (Proc. Seventh Conf., Kozubnik, 1979), pp. 18-67, Lecture Notes in Math., 798, Springer, Berlin-New York, 1980.

[2] K. S. Chang, D. H. Cho, and I. Yoo, Evaluation formulas for a conditional Feynman integral over Wiener paths in abstract Wiener space, Czechoslovak Math. J. 54(129) (2004), no. 1, 161-180.

[3] D. H. Cho, Conditional analytic Feynman integral over product space of Wiener paths in abstract Wiener space, Rocky Mountain J. Math. 38 (2008), no. 1, 61-90.

[4] _ A simple formula for an analogue of conditional Wiener integrals and its applications, Trans. Amer. Math. Soc. 360 (2008), no. 7, 3795-3811.

[5] _ Conditional Feynman integral and Schrödinger integral equation on a function space, Bull. Aust. Math. Soc. 79 (2009), no. 1, 1-22.

[6] _ A simple formula for an analogue of conditional Wiener integrals and its applications. II, Czechoslovak Math. J. 59(134) (2009), no. 2, 431-452.

[7] M. K. Im and K. S. Ryu, An analogue of Wiener measure and its applications, J. Korean Math. Soc. 39 (2002), no. 5, 801-819.

[8] G. W. Johnson and M. L. Lapidus, Generalized Dyson series, generalized Feynman diagrams, the Feynman integral and Feynman's operational calculus, Mem. Amer. Math. Soc. 62 (1986), no. 351, vi+78 pp.

[9] G. W. Johnson and D. L. Skoug, Notes on the Feynman integral, III: the Schroedinger equation, Pacific J. Math. 105 (1983), no. 2, 321-358.

[10] G. Kallianpur and C. Bromley, Generalized Feynman integrals using analytic continuation in several complex variables, Stochastic analysis and applications, 217-267, Adv. Probab. Related Topics, 7, Dekker, New York, 1984.

[11] H. H. Kuo, Gaussian measures in Banach spaces, Lecture Notes in Mathematics 463, Springer-Verlag, Berlin-New York, 1975.

[12] C. Park and D. L. Skoug, A simple formula for conditional Wiener integrals with applications, Pacific J. Math. 135 (1988), no. 2, 381-394.

[13] K. S. Ryu and M. K. Im, A measure-valued analogue of Wiener measure and the measure-valued Feynman-Kac formula, Trans. Amer. Math. Soc. 354 (2002), no. 12, 4921-4951.

[14] J. Yeh, Inversion of conditional expectations, Pacific J. Math. 52 (1974), 631-640.

[15] _ Inversion of conditional Wiener integrals, Pacific J. Math. 59 (1975), no. 2, $623-638$

[16] - Transformation of conditional Wiener integrals under translation and the Cameron-Martin translation theorem, Tôhoku Math. J. (2) 30 (1978), no. 4, 505-515.

Department of Mathematics

KYONGGI UNIVERSITY

SUWON 443-760, KoreA

E-mail address: j94385@kyonggi.ac.kr 